segregation error of maternal reciprocal translocation $t(9 ; 13)$ (p21;q12).

Conclusion Balanced reciprocal translocations in either parents can amplify and produce unbalanced gamets leading to defective conceptus. Prenatal diagnosis is strongly recommended where balanced translocation is found in parent. Clinical features of the affected conceptus depends largely on the regions of chromosome involved.

\section{P573 A CASE OF INSIDIOUS RECURRENT ABDOMINAL PAIN}

${ }^{1}$ Anita Spirito, ${ }^{1,2,3}$ Enrica Manca*, ${ }^{4}$ Claudio Carmine Guida, ${ }^{1}$ Angela Maggio, ${ }^{5}$ Maria Savino, ${ }^{4}$ Filippo Aucella, ${ }^{3}$ Massimo Pettoello-Mantovani, ${ }^{1}$ Saverio Ladogana. 'Department of Pediatrics, Onco-Hematology Unit, 'Casa Sollievo della Sofferenza' Scientific Institute, San Giovanni Rotondo, Foggia, Italy; ${ }^{2}$ Residency Program in Pediatrics, University of Foggia, Foggia, Italy; ${ }^{3}$ Department of Pediatrics, Pediatric Unit, 'Casa Sollievo della Sofferenza' Scientific Institute, San Giovanni Rotondo, Foggia, Italy; ${ }^{4}$ Department of Nephrology and Dialysis- Interregional Reference Center for the Prevention, Surveillance, Diagnosis and Treatment of Porphyria, 'Casa Sollievo della Sofferenza' Scientific Institute, San Giovanni Rotondo, Foggia, Italy; ${ }^{5}$ mmmunogenetic Laboratory- Department of Transfusion Medicine and Analysis Laboratory, 'Casa Sollievo della Sofferenza' Scientific Institute, San Giovanni Rotondo, Foggia, Italy

10.1136/archdischild-2019-epa.907

Porphyrias are a group of inherited metabolic disorders of heme biosynthesis leading to excessive accumulation and excretion of porphyrins.The variable clinical manifestations may determine a delay in the diagnosis, followed by a possible negative clinical outcome.

We describe a case of a 14-year-old girl with ShwachmanDiamond syndrome (SDS) and epilepsy admitted with severe cyclic abdominal pain not responsive to antalgic therapy, localized in the epigastric area, irradiating to the whole abdomen and the back, associated to aspecific symptoms (diarrhea, fever,vomiting). During hospitalization, the girl was asymptomatic. We excluded infectious, autoimmune, endocrinological causes, a relapse of SDS and performed a screening for porphyria that resulted negative. After each attack, she referred presenting urinary retention followed by hyperchromic urines, anxiety and paresthesias. Past clinical data showed hyponatremia, tachycardia and hypertension. Eventually, increased values of ALA $(8.21 \mathrm{mg} / \mathrm{l})$ and PBG $(3.96 \mathrm{mg} / \mathrm{l})$ were found; exposure of fresh urines to sunlight caused a change of their color. Finally, genetic analysis was negative. These findings allowed the diagnosis of porphyria, therefore we prescribed a normocalorichyperglucidic diet andpreventive glucose solutions in stressing situations, with regression of symptoms.

Acute porphyrias present with life-threatening crisis secondary to the injury of central, peripheral and autonomic nervous system. They can be triggered by drugs, alcohol, infections, reduced caloric intake, endogenous hormone cycles and stressing situations. The characteristic manifestations are severe cyclic abdominal pain, neurological or psychiatric symptoms and/or hyponatremia. Diagnostic is the assessment of plasmatic PBG/ALA, always increased during an attack, normal during remission. Treatment is human haemin although, in mild attacks, a diet with high carbohydrates and/or preventive glucose infusions is effective; a negative genetic evaluation should never exclude the diagnosis.

It is important considering acute porphyria in the differential diagnosis of severe cyclic abdominal pain, particularly in subjects affected by different and/or rare clinical disorders.

\section{P574 COMPLEX REGIONAL PAIN SYNDROME AND HPV VACCINE: A CASE REPORT OF COMPLEX REGIONAL PAIN SYNDROME AND LITERATURE REVIEW}

Isabela Govor*, Gerry Mackin, Dragos Valceanu. South West Acute Hospital, Enniskillen, UK

\subsection{6/archdischild-2019-epa.908}

Introduction Complex Regional Pain Syndrome (CRPS) is a chronic pain disorder characterised by significant autonomic features which typically develops in an extremity after acute tissue trauma. Symptoms may include continuing pain, sensory abnormalities like allodynia,hyperalgesia,hypoesthesia,vasomotor abnormalities in skin colour or temperature, sudomotor abnormalities of sweating or oedema and motor/trophic abnormalities in hair,skin,nails, tremor or muscle weakness.HPV vaccination is offered to all girls il Northern Ireland aged 14/ 15 years and recent plans have been made to offer it to boys starting late 2019. Since the introduction of HPV vaccination there have been case reports across the world describing various side effects after HPV vaccination, including Complex Regional Payn Syndrome. One report alone detailed 45 patients across 13 countries. In Japan the vaccination was temporarily halted as a consequence. The VaccineAdverseEventReportingSystem(VAERS) USA, has received 31.911 reports of adverse events following any HPV vaccination from which 22 $(0.07 \%)$ reports met the criteria for complex regional pain syndrome (including reflex sympathetic dystrophy) as an adverse event. 21 cases were after Gardasil (HPV4) vaccine and 1 was after Cervarix (HPV2) vaccine.

Case We report a previously fit healthy 14 years old Irish girl. She had received her 2nd HPV (Gardasil) vaccine on 09/04/ 18 having received her 1 st vaccine without any problems in October 2017. Twenty minutes post vaccine her left arm from the elbow down became swollen, purple and painful.Presentation was consistent, CRPS which was initially not diagnosed at first presentation to ED and Paediatrics. Correct diagnosis made four weeks post event and appropriate treatment initiated with full recovery withing eight months.

Laboratory investigations No significant abnormalities.

Conclusion There are documented cases of CRPS after HPV vaccine although is still controversial as to whether it is a vaccine or the effect of the trauma associated with inserting a needle which is the aetiological factor. This case highlights the need for better recognition of this condition amongst paediatricians as earlier initiation of treatment is associated with better long term outcome.

\section{P575 CASE REPORT: TWINS WITH NIEMAN PICK SYNDROME}

Samy AA Allawendy*, Cormac Duff, Dara Gallagher, Ann Sheehan. Sligo University Hospital, Sligo, Ireland

\subsection{6/archdischild-2019-epa.909}

Introduction Niemann-Pick Disease is an autosomal recessive metabolic disorder characterized by deposition of sphingomyelin and cholesterol within the lysosome of reticuloendothelial cells of various organs due to inherited deficiency of an enzyme, acid sphingomyelinase. Niemann-Pick Disease is classified into four types such as A, B, C and D each caused by a different gene mutation.

Case We report a case of Niemann Pick Disease type A/B twins. The patients are 3 years old girls, who presented to Sligo University Hospital with repeated episodes of chest 